C-A/AP/\#309

May 2008

\title{
RHIC RF Harmonic Numbers For Low Energy Operations
}

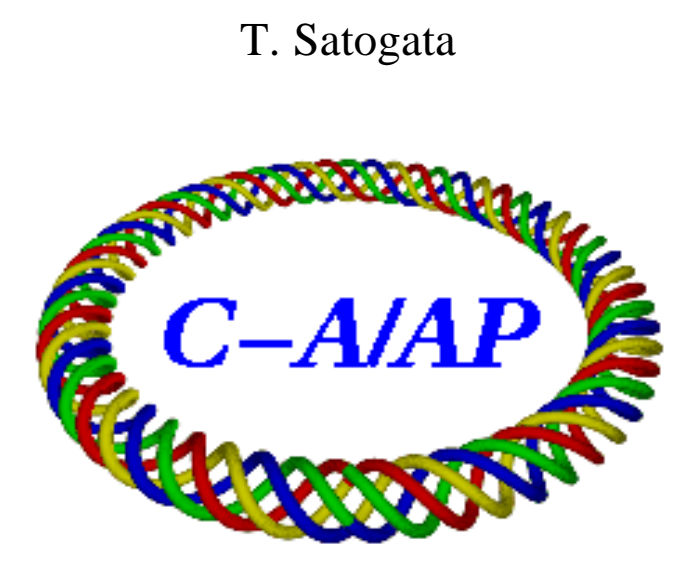

\section{Collider-Accelerator Department Brookhaven National Laboratory Upton, NY 11973}

Notice: This document has been authorized by employees of Brookhaven Science Associates, LLC under Contract No. DE-AC02-98CH10886 with the U.S. Department of Energy. The United States Government retains a non-exclusive, paidup, irrevocable, world-wide license to publish or reproduce the published form of this document, or allow others to do so, for United States Government purposes. 


\title{
RHIC RF Harmonic Numbers For Low Energy Operations
}

\author{
T. Satogata
}

May 6, 2008

\begin{abstract}
There have been several test runs of RHIC operations to explore the feasibility of luminosity production at low energies. There is considerable international interest in the possible existence of a QCD phase diagram critical point in the RHIC gold-gold collision energy range of $\sqrt{s_{\mathrm{NN}}}=$ $5-50 \mathrm{GeV}[1,2,3]$. This paper reviews the RF harmonic number constraints for RHIC goldgold collisions in this energy range, and concludes that optimal simultaneous collisions at both experiments are only feasible when the harmonic number is divisible by 9 .
\end{abstract}

\section{Harmonic Number Constraints}

RHIC has two RF systems that operate at frequencies of about $28 \mathrm{MHz}$ (injection and acceleration) and $7 \times 28=196 \mathrm{MHz}$ (storage). For low energy operations, typical total bunch lengths of 8$30 \mathrm{~ns}$ are much longer than the storage RF wavelength of $5 \mathrm{~ns}$, so the storage RF is not used. In normal operations, the $28 \mathrm{MHz}$ system frequency is defined to be exactly 360 times the beam revolution frequency. This factor of 360 is called the RF harmonic number $h$. For the nominal RHIC circumference $C_{\mathrm{RHIC}}=3833.845 \mathrm{~m}$ and $\mathrm{Au}$ injection total beam energy of $E=9.80 \mathrm{GeV} /$ nucleon $(\beta=0.99547)$, the revolution period is $t_{\mathrm{rev}}=12.84 \mu \mathrm{s}$, and the RF frequency is $f_{\mathrm{rf}}=28.023 \mathrm{MHz}$.

The acceptable tuning range of the RHIC $28 \mathrm{MHz}$ RF cavities is $f_{\mathrm{rf}}=28.0-28.17 \mathrm{MHz}$ [4]. As the beam energy is lowered, the revolution frequency and RF frequency drop. Below $E=9.01 \mathrm{GeV}$ the harmonic number must be raised to keep the RF cavities within their tuning range. At the lowest energy of interest, $E=2.5 \mathrm{GeV} /$ nucleon, $h=387$ gives an accessible RF frequency of $f_{\mathrm{rf}}=28.0847 \mathrm{MHz}$. A summary of RHIC harmonic numbers and permissible energy ranges based on the RF tuning constraint is given in Table 1 .

\begin{tabular}{|c|c|c|c|}
\hline $\mathrm{n} \mathrm{h}$ & Allowed $\sqrt{s_{N N}}[\mathrm{GeV}]$ & $h \bmod (3)=0$ & $h \bmod (9)=0$ \\
\hline 360 & $18.0-107$ & $*$ & $*$ \\
363 & $11.34-15.15$ & $*$ & \\
366 & $9.0-10.55$ & $*$ & $*$ \\
369 & $7.71-8.60$ & $*$ & $*$ \\
372 & $6.87-7.47$ & $*$ & \\
375 & $6.27-6.71$ & $*$ & $*$ \\
378 & $5.81-6.15$ & $*$ & \\
381 & $5.45-5.72$ & $*$ & \\
384 & $5.15-5.38$ & $*$ & $*$ \\
387 & $4.91-5.10$ & $*$ & \\
\hline
\end{tabular}

Table 1: Harmonic number choices for various collision energy ranges in the RHIC Low Energy program. These correspond to the acceptable RF frequency range of 28.0-28.17 MHz as described in the text. Operations at other harmonic numbers reduce trigger luminosity by a factor of three due to precessing of experiment trigger clocks.

The STAR and PHENIX experiment trigger clocks further constrain the harmonic number. The experiments currently require periodic clocks triggered every third bucket, or at the frequency $h f_{\mathrm{rf}} / 3$. If $h \bmod (3) \neq 0$ (i.e. the harmonic number is divisible by 3 with no remainder), the experiment clocks will precess from turn to turn; this reduces their usable luminosity by a factor of three. We therefore at least require that $h \bmod (3)=0$, as shown in Table 1 . Scenarios in which this constraint is loosened are described in the next section. 
Since PHENIX and STAR are separated by 60 degrees of the RHIC circumference (so colliding bunch patterns are separated by 120 degrees), it was originally thought that the $h \bmod (3)=0$ condition would be sufficient to imply collisions of both beams at both experiments. However, this is not the case.

To demonstrate this, we enumerate RHIC buckets in each ring as $0-(h-1)$, instead of the usual convention of $1-h$; the experiment trigger clock condition constrains usable beam to buckets $3 k$ where $k$ is an integer. Assume without loss of generality that blue bucket $3 k_{b}$ collides with yellow bucket $3 k_{y}$ at STAR. Then the same blue bucket $3 k_{b}$ collides with yellow bucket $\left(3 k_{y}+h / 3\right) \bmod (\mathrm{h})$ at PHENIX. Since $h \bmod (3)=0$, the only way that this yellow bucket can contain beam that is acceptable to experiment triggers is if $(h / 3) \bmod (3)=0$ or $h \bmod (9)=0$. Without this condition, each bunch can collide only once per turn at either STAR or PHENIX.

This constraint was a limiting factor in the 2008 low energy test run at $\sqrt{s_{N N}}=9.2 \mathrm{GeV}$, which used $h=366$. Simultaneous collisions for both experiments were not feasible, and each store instead cogged all collisions at either STAR or PHENIX.

There are other fill patterns with harmonic numbers not divisible by 9 that distribute low energy collisions between both experiments. However, all of these suboptimally redistribute collisions, do not create conditions where any bunches encounter collisions at both experiments, and do not increase the overall facility delivered luminosity. This operating condition would also require a change to the beam sync clock configurations so PHENIX and STAR would have different clock phases with respect to bunch crossings at 4 o'clock.

\section{Alternative Trigger Scenarios}

The $h \bmod (9)=0$ constraint comes from two sources: experiment trigger clocks and the geometrical phase between STAR and PHENIX. The latter would be prohibitively costly to change, requiring a chicane that lengthens the distance between STAR and PHENIX by at least one RF bucket.

What are the implications of loosening the experiment trigger clock constraint? This would permit beam in any bucket, and allow experiment triggers on collisions of any two buckets rather than only those divisble by 3 . A simple near-optimal fill pattern is shown in Table 2, where successive bunches are spaced by at least three buckets due to injection kicker constraints.

\begin{tabular}{|c|c|}
\hline STAR & PHENIX \\
\hline $1-1$ & $1-123$ \\
$123-123$ & $123-245$ \\
$245-245$ & $245-(2)$ \\
$4-4$ & $4-126$ \\
$126-126$ & $126-248$ \\
$248-248$ & $248-(5)$ \\
$7-7$ & $7-129$ \\
$129-129$ & $129-251$ \\
$251-251$ & $251-(8)$ \\
$10-10$ & $10-132$ \\
$132-132$ & $132-254$ \\
$254-254$ & $254-(11)$ \\
$\ldots$ & $\ldots$ \\
\hline
\end{tabular}

Table 2: A sample fill pattern for $h=366$ after eliminating the $h \bmod (3)=0$ experiment trigger clock constraint. Numbers shown are colliding buckets in the blue and yellow rings, respectively. This fill pattern produces full luminosity at STAR and about $2 / 3$ of full luminosity at PHENIX. Unfilled buckets colliding with filled buckets in PHENIX are shown in parentheses.

Collision clocking with this fill pattern requires either clocking every bucket or clocking asynchronously on every bunch crossing. Clocking on every bucket is infeasible, as it is too fast for existing experiment DAQs. Experiment DAQ experts are evaluating the feasibility of asynchronous clocking by bypassing DAQ PLLs, which currently halt runs whenever there is a clock phase jump and loss of PLL lock. 


\section{Conclusions}

The tuning constraints of the RHIC RF, combined with STAR and PHENIX trigger constraints, directly impact the RHIC low energy program. Luminosities are reduced by a factor of three for collision energies with harmonic numbers not divisible by three, such as $\sqrt{s_{\mathrm{NN}}}=15.15-18.0 \mathrm{GeV}$. Fortunately these "blind spots" get smaller at lower energies.

A stronger constraint arises from the combination of experiment triggers and geometry of the RHIC experiments. This constraint limits optimal collisions at both experiments only when the harmonic number is divisible by 9, or in CM energy ranges of $\sqrt{s_{\mathrm{NN}}}=4.91-5.10 \mathrm{GeV}, 5.81-$ $6.15 \mathrm{GeV}, 7.71-8.60 \mathrm{GeV}$, and above $18 \mathrm{GeV}$. Elimination of the experiment trigger constraint would allow full luminosity at one experiment and simultaneous $2 / 3$ of full luminosity at the other experiment for all permissible energies.

\section{References}

[1] M. Stephanov, K. Rajagopal, and E. Shuryak, "Signatures of the Tricritical Point in QCD", Phys. Rev. Lett. 81, 4816-4819 (1998).

[2] RIKEN workshop proceedings, "Can We Discover the QCD Critical Point at RHIC", March 9-10 2006, https://www.bnl.gov/riken/QCDRhic/.

[3] G.S.F. Stephans, "critRHIC: the RHIC low energy program", J. Phys. G: Nucl. Part. Phys. 32 (2006) S447-S453.

[4] A. Zaltsman, private communication. 